\title{
Outcome of Treatment with Thalidomide in Transfusion Dependent Thalassemia Patients: A Prospective Study in a Thalassemia Center, Dhaka, Bangladesh
}

\author{
Maksuda Begum ${ }^{1, ~ *, ~ M i r ~ H a s a n ~ M d . ~ M o s l e m ¹, ~ N u r u n ~ N a h a r ~ F a t e m a ~ B e g u m, ~}$ \\ Md. Zobaidur Rahman ${ }^{2}$ \\ ${ }^{1}$ Department of Pediatrics, Combined Military Hospital, Dhaka, Bangladesh \\ ${ }^{2}$ Armed Forces Medical Institute, Dhaka Cantonment, Dhaka, Bangladesh
}

Email address:

maksuda.begum343@gmail.com (M. Begum)

${ }^{*}$ Corresponding author

\section{To cite this article:}

Maksuda Begum, Mir Hasan Md. Moslem, Nurun Nahar Fatema Begum, Md. Zobaidur Rahman. Outcome of Treatment with Thalidomide in Transfusion Dependent Thalassemia Patients: A Prospective Study in a Thalassemia Center, Dhaka, Bangladesh. American Journal of Pediatrics. Vol. 6, No. 3, 2020, pp. 168-171. doi: 10.11648/j.ajp.20200603.11

Received: March 15, 2020; Accepted: March 27, 2020; Published: April 30, 2020

\begin{abstract}
The aim of the study was to assess the outcome of thalidomide in patients with Thalassemia. Method: We identified the outcome of thalidomide in 51 patients with thalassemia needing blood transfusion. We enlisted thalassemia patients, who received treatment with thalidomide between 01 July 2017 and 29 Feb 2020 in Combined Military Hospital, Dhaka. Thalidomide was used in a reduced dose $(2 \mathrm{mg} / \mathrm{kg}$ to $5 \mathrm{mg} / \mathrm{kg})$. Thalidomide had been stopped in those patients who developed complications. The following factors were recorded: age and sex, hemoglobin ( $\mathrm{Hb})$ levels, ferritin level, blood transfusion interval before and after treatment with thalidomide. Furthermore, we documented blood transfusion dependence and the complications subsequently. All data were studied using SPSS v 24.0 (SPSS Inc. Chicago, IL, U.S.A.). For patients, a paired sample t-test was used to compare the changes in the mean $\mathrm{Hb}$ level, ferritin level and the difference in blood transfusion interval before and after treatment with thalidomide. $\mathrm{P}<0.05$ was considered statistically significant. Results: The study included 51 patients comprised of 23 females (45.1\%) and 28 males (54.9\%) with an age ranging from 3 to 24 years $(9.75 \pm 4.11$ years). Amongst them $18 \%(n=9)$ were beta-thalassemia and $82 \%(n=42)$ were Hb E betathalassemia. Data of $\mathrm{Hb}$ and ferritin levels before and after treatment were recorded in all 51 patients. Ferritin levels reduced to $20.52 \%(3258.11 \pm 2291.91 \mathrm{ng} / \mathrm{ml}$ to $2589.65 \pm 2072.74 \mathrm{ng} / \mathrm{ml})$. Hb level increased by $19.26 \%(7.04 \pm 0.53 \mathrm{~g} / \mathrm{dL}$ to $8.39 \pm 1.44 \mathrm{~g} / \mathrm{dL})$. We found $18(35.3 \%)$ patients with no requirement of blood transfusion after the treatment. $63 \%(\mathrm{n}=32)$ of the patients showed no adverse effect of thalidomide after the treatment. $16 \%(\mathrm{n}=8)$ of the patients showed high ALT (Alaline Aminotransferase) and 6\% $(\mathrm{n}=3)$ showed excessive sleepiness. Other effects included- high TSH, acute urticaria, restlessness, edema, facial puffiness, palpitation, and vomiting. Conclusion: Treatment with thalidomide considerably improved $\mathrm{Hb}$ level, increased blood transfusion interval even resulted to non-dependence on blood transfusion and reduced ferritin levels. More studies are necessary to clarify the possible use of thalidomide in the treatment of thalassemia.
\end{abstract}

Keywords: Thalidomide, Transfusion, Thalassemia

\section{Introduction}

Thalassemia is recognized as a genetic condition of blood defined by reduced or deficiency of the globin chain synthesis. A person in such state is incapable to produce normal oxygen-carrying components of blood. [1] Thalassemia was first identified in the Mediterranean population. This condition is more pronounced in the thalassemia belt region of the world. Around 15 million population is suffering from this condition and about 240 million population are reported as the carrier. In India only the thalassemia carrier ranges from $1 \%$ to $17 \%$ (average of $3.2 \%)$ i.e. 1 in every 25 people in India are the carrier of thalassemia. [2, 3] Beta thalassemia is a type of thalassemia, 
in which Beta globin chain is compromised. E Beta thalassemia is a type in which $\mathrm{Hb} \mathrm{E}$ is produced alongside with the compromised beta chain. Apoptosis of cell is a result of excess development of $\alpha$-chain which initiates during erythropoiesis and reaches maximum concentration at polychromatophilic erythroblasts. [4]

Thalassemia has a limited treatment option. Management of thalassemia is channeled via treatment of severe anemia, prevention of excess erythropoiesis and iron overloading. [5, 6] In thalassemia blood transfusion is the mainstay of treatment at different frequency. Although few patients develop antibodies resulting into untransfusable condition and iron overloading causing damage to vital organs. Therefore, chelation therapy is mandatory in those circumstances along with single or in combination. Renal tubular damage, including Fanconi's Syndrome, has been documented in patients treated with deferasirox (one of the iron chelator), most likely in children and adolescents with beta-thalassemia and serum ferritin levels $<1500 \mathrm{mcg} / \mathrm{L}$. [7]

Though various options for management of thalassemia are available currently, yet there are limitations with the existing therapies, hence there is an unmet need for newer agents. Thalidomide, an old drug used for multiple myeloma treatment has appropriate effects in $\mathrm{Hb} \mathrm{F}$ induction with a similar mechanism of action with other immunomodulatory agents. The exact mechanism of the therapeutic effect of this factor is still unknown. However, the effects of thalidomide may be due to suppression of NF-KB induction by inflammatory cytokines such as Tumor Necrosis Factor (TNF- $\alpha$ ), Vascular Endothelial Growth Factor (VEGF) and prostaglandin E2 synthesis (PG-E2) associated with increased release of reactive oxygen species (ROS). ROS can launch P38 MAPK, which results in increased $\mathrm{Hb} F$ levels. The major side effects of thalidomide were reported as somnolence, Constipation, Gynecomastia, DVT. Adult patients were advised to avoid pregnancy during treatment. [8]

Presently there is no published study in Bangladesh on the outcome of thalidomide in thalassemia. This study will help to evaluate the outcome of thalidomide in thalassemia on the population of this country. This study also will help the future researcher in this field and will help in the treatment of thalassemia to reduce the frequency of blood transfusion even to be non-transfusion-dependent.

\section{Objectives}

General:

1. To find out the outcome of thalidomide in thalassemia patient.

Specific:

1. To find out the level of $\mathrm{Hb}$ after treatment with thalidomide.

2. To find out the transfusion interval after treatment with thalidomide.

3. To see the serum ferritin level after treatment with thalidomide.
4. To see the complications of treatment with thalidomide in patients of thalassemia.

\section{Materials and Methods}

A prospective study was conducted to evaluate the outcome of treatment with thalidomide in transfusion dependent thalassemia patients. We enlisted thalassemia patients who were on regular blood transfusion from the thalassemia center of combined military hospital Dhaka. Informed written permission had been taken from all the patient. After enrollment thalidomide had been started in a dose of $2 \mathrm{mg} / \mathrm{kg}$ to $5 \mathrm{mg} / \mathrm{kg}$. [9] Study duration was July 2017 to February 2020. After starting thalidomide patients were followed up regularly. Patients who received thalidomide for less than 03 months were not included in the study. Thalidomide was stopped in those patients who showed signs of complications. The following factors were recorded: age and sex, hemoglobin $(\mathrm{Hb})$ levels, ferritin level, blood transfusion interval, before and after treatment with thalidomide. Additionally, we recorded non-transfusiondependent and the complication after the treatment with thalidomide. Finally data were compiled and analyzed.

Inclusion criteria:

All patients of thalassemia who were taking regular blood transfusion from the thalassemia center of combined military hospital Dhaka were included in the study.

Exclusion criteria:

1. Those patients who were not eligible for taking thalidomide (Altered liver function, altered renal function, thrombotic predisposition), and who had lack of adherence to treatment.

2. Patients who received thalidomide for less than 03 months.

Statistical analysis

All data were analyzed with SPSS v 24.0 (SPSS Inc. Chicago, IL, U.S.A.). For patients, a paired test was conducted to evaluate the changes in the mean $\mathrm{Hb}$ level, ferritin level and the variance in blood transfusion interval before and after treatment with thalidomide. $\mathrm{P}<0.05$ was considered statistically significant. Pie charts and bar charts were used to present the gender distribution and blood transfusion interval days between non-transfusion- dependent and transfusion-dependent patients. Data on the types of complications and non-transfusion dependent after the treatment with thalidomide were presented as the percentage of the total number of observations.

\section{Results}

This study includes 51 patients, including 28 males and 23 females. The male to female ratio was high (1.22: 1). (Figure 1) The age of patients range from 3 years to 24 years $(9.75 \pm 4.11$ years). The highest number of patients belongs to the age of 6 -10-year-old (54.9\%) followed by 11 - 15 -year-old (27.5\%). (Table 1) Hemoglobin and serum ferritin levels and blood transfusion intervals have been measured in all patients 
before and after treatment with thalidomide. Initially, blood transfusion interval ranged from 15 days to 3 months (33.02 \pm 13.02 days), whereas after the treatment it ranges from 25 days to 2 years $(129 \pm 137.31$ days). There is a statistically significant difference $(p$-value $<.0001)$. (Table 2)

Before treatment, hemoglobin was ranged from $5 \mathrm{~g} / \mathrm{dL}$ to 8 $\mathrm{g} / \mathrm{dL}(7.04 \pm 0.53 \mathrm{~g} / \mathrm{dL})$, while after treatment with thalidomide, it range has increased from $5 \mathrm{~g} / \mathrm{dL}$ to $12 \mathrm{~g} / \mathrm{dL}$ $(8.39 \pm 1.44 \mathrm{~g} / \mathrm{dL})$ There is a statistically significant difference (p-value $=0.001)$. (Table 2)

Before treatment, serum ferritin was $300 \mathrm{ng} / \mathrm{mL}$ to 9000 $\mathrm{ng} / \mathrm{mL}(3258.11 \pm 2291.91 \mathrm{ng} / \mathrm{mL})$, while after treatment, it decreased from $400 \mathrm{ng} / \mathrm{mL}$ to $10000 \mathrm{ng} / \mathrm{mL}$ $(2589.65 \pm 2072.74 \mathrm{ng} / \mathrm{mL})$. The difference was statistically significant ( $\mathrm{p}$-value $=0.003$ ). Clinical features of patients are described (Table 3). All patients show different transfusion interval. (Table 2). Presently there is no blood transfusion required for $35.3 \%(\mathrm{n}=18)$ of the patients after the treatment. (Table 3). 11 (22\%) patients' have no change in the blood transfusion interval and 22 patients require blood transfusion but their transfusion interval has significantly increased. Among them, $3(6 \%)$ patients require a blood transfusion after 5 months of interval and $3(6 \%)$ patients require a blood transfusion after 8 months of interval. (Figure 2). Sixty three $(63 \%)$ of the patients show no adverse effect of thalidomide after the treatment. However, $16 \%(n=8)$ of the patients show high ALT (Alanine Aminotransferase) and 6\% $(n=3)$ show excessive sleepiness. Other adverse effects included- high TSH, acute urticaria, restlessness, edema, facial puffiness, palpitation and vomiting. (Table 4)

Table 1. Age Distribution of the patients $(n=51)$.

\begin{tabular}{lll}
\hline Age & Number of Patients (Percentage) & Mean \pm SD \\
\hline 0 to 5 & $5(9.8 \%)$ & \\
6 to 10 & $28(54.9 \%)$ & \\
11 to 15 & $14(27.5 \%)$ & $9.75 \pm 4.11$ \\
16 to 20 & $2(3.9 \%)$ & \\
21 to 25 & $2(3.9 \%)$ & \\
Total & $51(100 \%)$ & \\
\hline
\end{tabular}

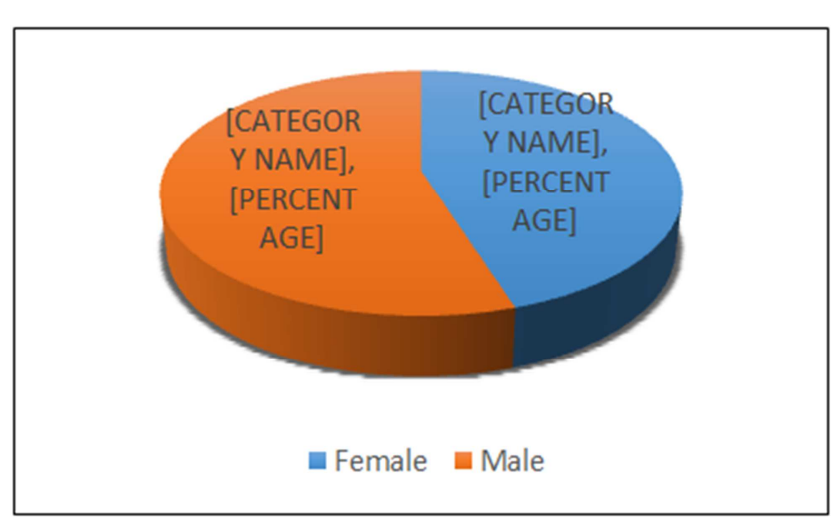

Figure 1. Gender Distribution $(n=51)$.

Table 2. Difference in BT interval, hemoglobin and ferritin levels before and after treatment ( $n=51)$.

\begin{tabular}{lll}
\hline & Min, Max & Mean \pm S. D \\
\hline Previous BT interval & 15 days, 90 days (3month) & $33.02 \pm 13.02$ \\
Present BT interval & 25 days, 730 days (2 years) & $129.1 \pm 137.31$ \\
Previous Hb & 5,8 & $7.04 \pm 0.53$ \\
Present Hb & 5,12 & $8.39 \pm 1.44$ \\
Previous Ferritin level & 300,9000 & $3258.11 \pm 2291.91$ \\
Present Ferritin level & 400,10000 & $2589.65 \pm 2072.74$ \\
\hline
\end{tabular}

Table 3. Blood Transfusion Requirement $(n=51)$.

\begin{tabular}{ll}
\hline Blood Transfusion Requirement & Number of Patients (Percentage) \\
\hline Yes & $33(64.7 \%)$ \\
No & $18(35.3 \%)$ \\
Total & $51(100 \%)$ \\
\hline
\end{tabular}

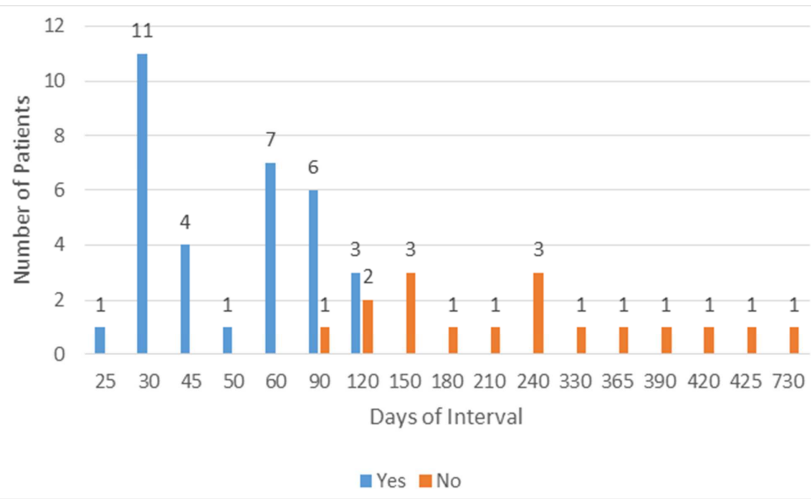

Figure 2. Days of interval of blood transfusion $(n=51)$.
Table 4. Side effects of Thalidomide $(n=51)$.

\begin{tabular}{ll}
\hline Side Effects & Number Patients (Percentage) \\
\hline No Side Effect & $32(63 \%)$ \\
High Serum ALT & $8(16 \%)$ \\
Excessive sleepiness & $3(6 \%)$ \\
High TSH & $2(4 \%)$ \\
Acute Urticaria & $1(2 \%)$ \\
Restlessness & $1(2 \%)$ \\
Edema, Facial puffiness & $1(2 \%)$ \\
Palpitation & $1(2 \%)$ \\
Cough & $1(2 \%)$ \\
Vomiting & $1(2 \%)$ \\
Total & $51(100 \%)$ \\
\hline
\end{tabular}

\section{Discussion}

Thalassemia is a result of defects in the globin gene. This shortage and the additional $\alpha$-globin chains result in oxidative stress, dyserythropoiesis, and chronic anemia. Thalassemia major results in severe anemia, poor growth, and skeletal defects and needs consistent, lifelong blood transfusions. Thalassemia intermedia consist of only recurrent blood transfusions, while 
Thalassemia minor does not need a specific treatment. The credible method to lessening the patients' burden is proficient prevention: carrier recognition, prenatal analysis, and genetic investigation. [10] Thalidomide is a drug known for its immunomodulation and anti-angiogenic properties. It has recently been stated to induce $\gamma$-globin gene expression and to escalate the increase of erythroid cells. [11, 12] In our study, we assessed the outcome of treating patients with thalidomide to understand its effect on hemoglobin and serum ferritin levels. It was found that mean hemoglobin levels were increased after treatment with thalidomide, while serum ferritin levels considerably decline after treating with thalidomide. It is strongly suggestive of the effect of thalidomide on $\mathrm{HbF}$ and also a reduction in the iron deposition. To date, not many studies were conducted on the effect of thalidomide. Fozza undertook 2 cases of non-transfusiondependent thalassemia. $\mathrm{HbF}$ was considerably elevated in these patients after treatment with low-dose thalidomide. [13] Ali conducted a study in 2016. Patients of $\beta$-thalassemia major were treated with thalidomide and sodium butyrate. He recommended that thalidomide was more efficient [14] However, Gunaseelan stated a case of stroke in a $\beta$-thalassemia child who was treated with a course of thalidomide. [15] Chen stated in a study that thalidomide showed an exceptional effect on $\beta$-thalassemia patients who required repetitive red-cell transfusions. Thalidomide improved hemoglobin levels without having any serious effect. [16]

\section{Conclusion}

Our prospective study suggests that thalidomide has a greater healing effect in patients with thalassemia. Thalidomide significantly increased the blood transfusion interval and thereby helps to reduce the ferritin level in most of the patients with very limited adverse effects. Additional multicenter prospective clinical trials are required to approve the effectiveness and safety of thalidomide in treating patients of transfusion-dependent thalassemia patients.

\section{Limitations of the Study}

This is a single Center study with small sample size. So, the study result may not reflect the scenario of the whole country.

\section{Approval}

Got approval from the respective department.

\section{Funding Source}

Self.

\section{Conflict of Interest}

Not declared.

\section{References}

[1] “Transport of Oxygen in the Blood.” (2016) Boundless Biology.

[2] Aggarwal R, Prakash A, Aggarwal M (2014) Thalassemia: An overview. J Scient Society 41: 3-6.

[3] Rund D, Rachmilewitz E (2005) $\beta$-Thalassemia. N Engl J Med 353: 1135-1146.

[4] Management of Haemoglobin Disorders (2008) Joint WHOTIF meeting on management of hemoglobin disorders [Monograph Online] Geneva, World Health Organization.

[5] Tripette J, Alexy T, Hardy-Dessources MD, Mougenel D, Beltan E, et al. (2009) Red blood cell aggregation, aggregate strength and oxygen transport potential of blood are abnormal in both homozygous sickle cell anemia and sickle-hemoglobin C disease. Haematologica 94: 1060-1065.

[6] Weiss MJ, dos Santos CO (2009) Chaperoning erythropoiesis. Blood 113: 2136-2144.

[7] Lemonne N, Charlot K, Waltz X, Ballas SK, Lamarre Y, et al. (2015) Hydroxyurea treatment does not increase blood viscosity and improves red blood cell rheology in sickle cell anemia. Haematologica 100: e383-386.

[8] Fard AD, Hosseini SA, Shahjahani M, Salari F, Jaseb K (2013) Evaluation of Novel Fetal Hemoglobin Inducer Drugs in Treatment of $\beta$-Hemoglobinopathy Disorders. Int J Hematol Oncol Stem Cell Res 7: 47-54.

[9] Ramanan. V, Kelkar. K. Sci Forschen Journal of Blood Disorders and Medicine Role of Thalidomide in Treatment of Beta. 2017: 8-10.

[10] Fibach E, Rachmilewitz EA. Pathophysiology and treatment of patients with beta-thalassemia - an update. F1000Research. 2017; 6 (0). doi: 10.12688/f1000research.12688.1).

[11] Aerbajinai W, Zhu J, Gao Z, et al. Thalidomide induces $\gamma$ globin gene expression through increased reactive oxygen species-mediated p38 MAPK signaling and histone $\mathrm{H} 4$ acetylation in adult erythropoiesis.

[12] Moutouh-de Parseval LA, Verhelle D, Glezer E, et al. Pomalidomide and lenalidomide regulate erythropoiesis and fetal haemoglobin production in human CD34+ cells. J Clin Invest. 2008; 118: 248-58.

[13] Fozza C, Pardini S, Giannico DB, et al. Dramatic erythroid response to low-dose thalidomide in two patients with transfusion independent thalassemia and severe post-transfusional alloimmune hemolysis. Am J Hematol. 2015; 90 (7): E141.

[14] Jalali Far MA, Dehghani Fard A, Hajizamani S, et al. Thalidomide is more efficient than sodium butyrate in enhancing GATA-1 and EKLF gene expression in erythroid progenitors derived from HSCs with $\beta$-globin gene mutation. Int J Hematol Oncol Stem Cell Res. 2016; 10 (1): 37-4.

[15] Gunaseelan. S and Anand Prakash. Thalidomide-induced Stroke in a Child With Thalassemia. Journal of Pediatric Hematology / Oncology: olters Kluwer Health, Inc. Date: Nov 1, 2017.

[16] Chen, J, Zhu, W, Cai, N, Bu, S, Li, J, Huang, L. Thalidomide induces haematologic responses in patients with $\beta$ thalassaemia. Eur J Haematol. 2017; 99: 437-441. https://doi.org/10.1111/ejh.12955). 